\title{
Transmission de données par les fournisseurs de prestations médicales aux organes de l'assurance-accidents obligatoire et de l'assurance militaire
}

Denise Rüegg

Directrice du Service central des tarifs médicaux LAA

Correspondance: Denise Rüegg Service central des tarifs médicaux LAA

Case postale 4358 CH-6002 Lucerne Tél. 0414195310
Dans son communiqué du 4 juillet 2012, l'Office fédéral de la santé publique (OFSP) a informé que les modifications de la loi sur l'assurance-maladie adoptées en décembre 2011 par le Parlement seront mises en vigueur par le Conseil fédéral le 1 1er janvier 2013. Celles-ci comprennent notamment une disposition réglant la transmission par les hôpitaux de fichiers de données aux assureurs-maladie (cf. art. 59a ss. OAMal, nouvelle version). A partir de 2014 au plus tard, les hôpitaux transmettront systématiquement, au moment de la facturation, les indications administratives et médicales à un service de réception des données certifié mis en place par l'assureur. Durant la période de transition, les indications médicales ne pourront être transmises systématiquement qu'au médecin-conseil. Le Conseil fédéral entend garantir ainsi la protection des patients.

L'OFSP communique que la disposition en vigueur à partir du $1^{\text {er }}$ janvier 2013 pour la transmission par les hôpitaux de fichiers de données dans le

\section{Factures DRG dans le domaine AA/AM/AI - commentaire de la FMH}

Selon la LAA, la LAM et la LAI, l'assureur a le droit d'obtenir et de traiter les données qui lui sont nécessaires [1] pour faire son travail correctement. Le Conseil fédéral décrit ainsi ce dont un assureur a besoin pour contrôler les factures DRG de manière correcte: «Afin de tenir compte du principe de proportionnalité, il faut prévoir une réglementation spécifique pour la réception et la transmission des données chez l'assureur. Pour cette raison, l'assureur doit disposer d'un «service de réception des données certifié». Un service de réception des données certifié a en particulier la tâche de procéder, par le biais d'un contrôle des factures entièrement automatisé, au triage des factures. Ce contrôle entièrement automatisé des factures consiste en un triage automatisé, dont l'objectif est de filtrer uniquement les «factures suspectes» pour un examen plus détaillé. Les factures filtrées sont transmises à l'assureur pour une clarification manuelle complémentaire. Toutes les autres factures sont libérées pour paiement, sans que les collaborateurs de l'assureur puissent avoir connaissance des indications médicales.» [2].

La fonction différente de l'assureur-maladie et de l'assureur-accident ne joue aucun rôle dans le contrôle des factures; il n'y a en effet aucune différence entre le contrôle des factures hospitalières établies selon le système du tiers payant et celles établies selon le principe des prestations en nature: un contrôle est un contrôle. Les exigences du Conseil fédéral s'appliquent donc par analogie à tous les répondants des coûts qui souhaitent obtenir ces informations, que ce soit aux assureurs AA/AM/Al ou aux cantons qui souhaitent contrôler eux-mêmes les factures DRG.

Dr Pierre-François Cuénoud, membre du Comité central de la FMH, responsable du domaine Tarifs et économie de la santé pour les médecins hospitaliers Hanspeter Kuhn, avocat, responsable du Service juridique de la FMH, secrétaire général adjoint

1 par ex. LAA

Art. 96 LAA, Traitement de données personnelles

Les organes chargés d'appliquer la présente loi, d'en contrôler ou surveiller l'exécution sont habilités à traiter et à faire traiter les données personnelles, y compris les données sensibles et les profils de la personnalité, qui leur sont nécessaires pour accomplir les tâches que leur assigne la présente loi, notamment pour:

a. calculer et percevoir les primes;

b. établir le droit aux prestations, les calculer, les allouer et les coordonner avec celles d'autres assurances sociales;

c. surveiller l'application des dispositions sur la prévention des accidents et des maladies professionnelles;

d. faire valoir une prétention récursoire contre le tiers responsable;

e. surveiller l'exécution de la présente loi;

f. établir des statistiques;

g. attribuer ou vérifier le numéro d'assuré AVS.

2 Commentaire sur la révision de l'OAMal 
domaine de l'assurance-maladie ne s'applique ni à l'assurance-accidents ni à l'assurance militaire. Dans ces deux derniers domaines, les données continueront donc d'être transmises directement par le fournisseur de prestations après le $1^{\text {er }}$ janvier 2013 (art. 54a LAA).

Dans son exposé des motifs, l'OFSP rappelle qu'il existe des différences entre l'assurance-maladie et approprié de l'assuré (art. 48 al. 1 LAA). Or, pour pouvoir prendre les mesures utiles à la détermination du traitement complet et approprié, l'assureur a besoin des données médicales nécessaires, qui sont intégralement déposées dans un dossier de patient.

En vertu de l'art. 96 al. 1 LAA, les organes chargés d'appliquer la loi sont habilités à traiter et à faire traiter les données personnelles, y compris les données

\section{Dans ces deux derniers domaines, les données continueront donc d'être transmises directement par le fournisseur de prestations après le 1 er janvier 2013.}

l'assurance-accidents. Dans l'assurance-accidents, c'est le principe des prestations en nature qui s'applique. En vertu de ce principe, l'assureur prend en charge un traitement complet et approprié du patient et n'assume pas uniquement, comme dans l'assurance-maladie, les coûts facturés dans le cas d'espèce (principe du remboursement des frais).

L'approche des prestations en nature permet notamment à l'assureur d'influer sur l'ampleur, la nature et la durée des prestations. L'assureur est ainsi habilité à prendre les mesures qu'exige le traitement sensibles et les profils de la personnalité, par exemple afin d'évaluer les droits aux prestations. Autrement dit, les dispositions de la LAA relatives à la protection des données exigent elles aussi la transmission directe et systématique des données des patients aux assureurs LAA. Il n'existe aucune restriction légale découlant des indications répertoriées dans l'article 96 LAA. Ces principes s'appliquent également aux domaines de l'assurance militaire et de l'assurance-invalidité.

\section{Vous qui lisez une revue des Editions médicales suisses,}

\section{saviez-vous que ...}

- les Editions médicales suisses sont une coopération entre la Fédération des médecins suisses (FMH) et la plus ancienne maison d'édition et imprimerie au monde (les Editions Schwabe, fondées en 1488)?

que les EMH sont les éditions de pointe en Suisse dans le domaine des journaux médicaux, avec dix revues spécialisées, des articles paraissant intégralement en ligne et un choix de livre de plus en plus large?

que toutes les revues paraissant aux EMH sont les organes officiels de publication des sociétés médicales correspondantes?

Si vous souhaitez en savoir plus sur les $\mathrm{EMH}$, vous trouverez plus d'informations sous www.emh.ch.

EMH Editions médicales suisses - des publications à la pointe de la médecine 